\title{
Rats acquire a low-response-cost daily time-place task with differential amounts of food
}

\author{
Christina M. Thorpe and Donald M. Wilkie \\ University of British Columbia, Vancouver, British Columbia, Canada
}

\begin{abstract}
Gallistel (1990) theorized that when animals encounter a biologically significant event, they automatically form a tripartite code consisting of the time, place, and nature of the event. Recent research examining such time-place learning (TPL) has shown that rats are reluctant to perform TPL tasks and appear to do so only under high-response-cost situations (Thorpe, Bates, \& Wilkie, 2003; Widman, Gordon, \& Timberlake, 2000). In the present study, we trained rats on a low-response-cost daily TPL task, in which the amount of food varied with the spatiotemporal contingencies. It was found that rats readily learned this task. We hypothesize that, rather than automatically encoding a tripartite code when faced with a biologically important event, rats instead automatically encode bipartite codes consisting of time-event and event-place information.
\end{abstract}

By looking at animals in both their natural environment and in the laboratory, one can see the importance that both temporal and spatial information exert over an animal's behavior. Gallistel (1990) noted the vital role of spatiotemporal knowledge and theorized that whenever a biologically significant event occurs, a memory code is automatically made that includes the nature of the event, the time of the event, and where it occurred. Later, the animal can consult these memory codes to determine when and where current needs have been met in the past, and it can use that information to guide its current behavior.

One of the reasons that spatiotemporal knowledge is so important is that many biologically significant events have some degree of spatiotemporal regularity. For example, the availability of food, mates, and predators varies predictably across both space and time. The ability to learn the spatiotemporal variability of stimuli is known as time-place learning (TPL). The present article will focus on one particular type of TPL: daily TPL. (For a discussion of other types of TPL, see Thorpe \& Wilkie, 2006).

Biebach, Gordijn, and Krebs (1989) provided the first laboratory demonstration of daily TPL. In their experiment, garden warblers were tested for $12 \mathrm{~h}$ each day in a chamber divided into a central living area and four surrounding compartments. Each compartment contained a grain dispenser, which provided grain intermittently for a specific 3 -h period every day. The warblers learned to visit the compartments at the appropriate times. Other laboratory studies have shown similar TPL in honeybees (Wahl, 1932; cited in Reebs, 1993), pigeons (Saksida \& Wilkie, 1994), and fish (inangas) (Reebs, 1999).

Recently, there has been some debate about the ability of rats to display daily TPL. Thorpe, Bates, and Wilkie (2003) attempted to demonstrate daily TPL in rats using a variety of tasks including a place preference task, a semiaversive water maze task, a radial arm maze task, and a T-maze task. They found that rats learned the locations of the food (or of the platform, in the case of the water maze task), as shown by their tendency to prefer those locations over others that had never been associated with food (or platforms). However, the rats did not associate those places with the time of day they were provided with food (or with a platform). Similar results were found on studies in which rats were trained to press one of two levers depending on the time of day (Boulos \& Logothetis, 1990). (See also unpublished data from Timberlake \& White, 1990, cited in Widman, Gordon, \& Timberlake, 2000.)

Means, Ginn, Arolfo, and Pence (2000) had some success in a T-maze version of the TPL task. Sixty-three percent of rats were able to learn this task, but only after significant training. However, with continued training, rats' performance actually decreased. In addition, when external time-related cues such as noises and feeding were minimized, the trials to criterion increased. In a later study, Means, Arolfo, Ginn, Pence, and Watson (2000) again showed that rats have difficulty learning a timeplace association in a T-maze. Neither making the two places more distinctive nor making the two times farther apart during the day significantly increased rats' success rate on the task.

Widman et al. (2000) discovered that, by increasing the response cost (i.e., effort) in TPL tasks, they increased the probability that the rats would learn the tasks. In a simple radial arm maze similar to those described above (i.e., one arm baited in morning sessions, another arm baited in afternoon sessions), the rats did learn a general place preference (i.e., they learned which two arms were baited), but they did not learn when those places were

C. M. Thorpe, christina.thorpe@ucl.ac.uk 
baited. By switching to a vertical maze and increasing the height the rats had to climb for food (and thereby increasing the response cost), they increased the success rate for TPL. Widman, Sermania, and Genismore (2004) showed a similar effect of response cost in a Morris water maze study.

There are, therefore, some obvious inconsistencies in the daily TPL literature. Some researchers have found daily TPL in operant response tasks (Carr \& Wilkie, 1997a, 1997b, 1999; Mistlberger, de Groot, Bossert, \& Marchant, 1996; Pizzo \& Crystal, 2002), whereas others using very similar paradigms and similar reinforcement schedules have failed to find it (Boulos \& Logothetis, 1990; Thorpe et al., 2003; White \& Timberlake, 1990). Although Widman et al. (2000) believe that response-cost ratios (the relative cost of making an error) are the critical determinants of whether an animal learns a time-place task, it is not clear from a theoretical perspective why this should be the case. Nor does the response-cost theory account for the many inconsistencies in the research findings.

It is also not clear whether the rats that are successfully learning these daily TPL tasks are using a circadian timing process. Carr and Wilkie (1997a) identified three timing systems: interval, ordinal, and phase. Interval timing allows an animal to anticipate the occurrence of an event that occurs a fixed amount of time after some external event. It is typically employed for durations ranging from milliseconds to several minutes, and thus is not applicable to the daily TPL tasks. Ordinal timing allows an animal to predict the order in which events occur within a particular time frame; for example, Carr and Wilkie (1997b) found that when rats trained on a daily TPL task were given probe tests in which the morning session was skipped, they responded in the afternoon as if it were the morning. When they were given probe tests in which the afternoon sessions were skipped, the rats went to the correct location the following morning. These skip probes suggest that the rats learned the order in which locations provided food within a given day, and that their timers were reset by some event sometime following the afternoon session. Means, Ginn, et al. (2000) found similar results in T-maze daily TPL tasks.

The third timing system is phase timing, the most often studied example of which is circadian timing. If a circadian timer is used, then-following probe tests in which either the morning or afternoon session is skipped - the animals should go to the correct location. In addition to these timing systems, it is important to rule out the use of the nontiming alternation strategy. Pizzo and Crystal (2004), using a radial arm maze TPL task, found that the rats responded incorrectly after both probe tests that skipped morning and afternoon sessions. This suggests the use of an alternation strategy.

In the present study, we hoped to demonstrate daily TPL in a low-response-cost task. Rats were trained in a standard daily TPL task that differed in one respect from past designs: The reward also differed depending on the time of day. For example, Place 1 provided a large amount of food at Time 1, whereas Place 2 provided a smaller amount of food at Time 2. Two dependent measures, the correctness of the choice and the latency to leave the start arm, were of interest. The latency to leave the start arm was taken as a measure of whether the rats had made an association between the time of day and the amount of food (assuming that the rats would have shorter latencies on trials in which they received more food than on trials in which they received less food). The correctness of the choice was used to determine whether the rats had learned the spatial component of the task. In addition to determining whether the rats would learn a low-response-cost daily TPL, we also hoped to determine whether learning was accomplished through the use of a circadian or ordinal timer, or through the use of a nontiming alternation strategy.

\section{METHOD}

\section{Subjects and Apparatus}

Seven male Long-Evans rats were obtained from Charles River (St. Constant, Quebec). At the start of the experiment, they were approximately 100 days old. They were maintained at approximately $90 \%$ of free-feeding weight, adjusted for age. They received a standard rat diet (PMI Feeds, Richmond, IN) at the end of test days (approximately 1,800 h), as well as on nontest days, to maintain their weight. During test sessions they received Froot Loops (FLs) (Kellogg Company, Battle Creek, MI). They had free access to water except during test sessions.

Rats were individually housed in transparent plastic cages lined with aspen woodchip bedding (Netco, New York, NY ). Every week, rats were given paper products with which to build nests. Rats received four to six sessions per week of group behavioral enrichment, which consisted of being placed in a chamber containing various tubes, ladders, toys, and other rats, for $20 \mathrm{~min}$. The behavioral sessions occurred during the light portion of the light-dark schedule and were held at various times of day, depending whether the enrichment chamber was available. The colony room was maintained on a 12:12-h light:dark cycle, with light onset at $0730 \mathrm{~h}$ and offset at $1930 \mathrm{~h}$.

The apparatus was an open, elevated, white wooden T-maze raised $70 \mathrm{~cm}$ above the floor. The stem and choice arms were $15 \mathrm{~cm}$ wide and $61 \mathrm{~cm}$ long. A circular 2-cm diameter food well was located at the end of each choice arm. Food wells were $0.5 \mathrm{~cm}$ deep. FLs were attached to the underside of the maze, out of reach of the rats, to mask the odor of the food reward in the maze. The maze was placed in a normally lighted room with several distal visual cues present, including a chair, boxes, cupboards, and two counters.

\section{Procedure}

Rats were trained twice daily, once in the morning from $0930 \mathrm{~h}$ to $1100 \mathrm{~h}$, and once in the afternoon from $1530 \mathrm{~h}$ to $1700 \mathrm{~h}$. Three rats were assigned one to the AM group (those who received one FL $[1 \mathrm{FL}]$ in the morning and one-fifth of a FL $[1 / 5 \mathrm{FL}]$ in the afternoon) and 4 rats were assigned to the PM group (those who received 1FL in the afternoon and $1 / 5 \mathrm{FL}$ in the morning). The location of the food differed depending on the time of day. For example, Rat 1 received $1 \mathrm{FL}$ in the left arm in morning sessions and $1 / 5 \mathrm{FL}$ in the right arm in afternoon sessions. The location of the morning and afternoon food was more or less counterbalanced across rats.

Each rat received three trials in each session. Each trial was separated by approximately 8 to $10 \mathrm{~min}$. For each trial, the rat was placed at the far end (i.e., the end not connected to the choice arms) of the start arm. Rats that did not exit the start arm after 2 min were removed from the maze. (This occurred rarely and only at the beginning of training.) If rats went to the incorrect arm, they were allowed to go into the correct arm to receive the food reward. Only data from the first trial of each session were considered, because Trials 2 and 3 were contaminated by Trial 1. For example, if on Trial 1 the rat received $1 \mathrm{FL}$ in the left arm, the rat was likely to return to that arm on 
subsequent trials within that session. Two variables were measured: the latency for the rats to leave the start arm and whether the first arm visited was the correct arm. Training occurred for 5-6 days per week and lasted for an average of 155 days.

Approximately every 2 weeks during training, the data from the previous 10 days were examined to determine whether the percentage of correct choices was above $75 \%$, and whether the latency on the $1 \mathrm{FL}$ trials was at least $2 \mathrm{sec}$ faster than the latency on the $1 / 5 \mathrm{FL}$ trials. When a rat met these criteria, it was deemed to have learned the task, and probe tests were begun to determine the timing strategy used. A maximum of one probe test was conducted per week. Probes were intermixed with baseline sessions. An average of 272 baseline sessions were completed from the end of training to the last probe test. On probe days, either the morning (Skip AM) session or the afternoon (Skip PM) session was skipped. Skip AM and Skip PM probes were given in a random order, which differed from rat to rat. The procedure was identical on nonprobe days, except that neither choice arm was baited and only one trial per session was performed. Rats were initially given 5 Skip AM and 5 Skip PM sessions each. However, upon inspection of the data, it was found that occasionally the rats' performance on the baseline sessions given between the probes had fallen below acceptable levels; that is, the percent of correct choices was less than $75 \%$ and the difference between AM and PM latencies was less than $2 \mathrm{sec}$. We chose to exclude probes in which the baseline data on the 4 days immediately preceding the probes were below these minimum requirements. The average number of probe trials excluded because they did not meet these minimum requirements was 2.7. Further probes were conducted until each rat had 3 Skip AM and 3 Skip PM probes. (Because Rat 3's baseline performance never returned to the required level to allow a final Skip AM session, the animal received only 2 Skip AM and 3 Skip PM probes.) Probes were only conducted if the rats met the minimum requirements listed above.

If rats were using an alternation strategy, they were expected to behave as though it were morning following a Skip AM session and as though it were afternoon following a Skip PM session. If rats were using circadian timing, their performance was expected to be unaffected by either Skip AM or Skip PM sessions. If rats were using ordinal timing, they were expected to behave as though it were morning following a Skip AM session, and to also behave as though it were morning following a Skip PM session. These possible outcomes are shown in Figure 1.

\section{RESULTS}

As discussed in the Method section, in all analyses only data from the first trial of each session were included. Figure 2 shows the average latency to leave the start arm and the percent of correct arm choices for the 10 days prior

\begin{tabular}{|c|c|c|}
\hline Mechanism & Skip PM (tested in AM) & Skip AM (tested in PM) \\
\hline Circadian & $\begin{array}{c}\text { Latency: similar to AM } \\
\text { Percent correct: } ~ 100 \%\end{array}$ & $\begin{array}{c}\text { Latency: similar to PM } \\
\text { Percent correct: } ~ 100 \%\end{array}$ \\
\hline Ordinal & $\begin{array}{c}\text { Latency: similar to AM } \\
\text { Percent correct: } ~ 100 \%\end{array}$ & $\begin{array}{c}\text { Latency: similar to AM } \\
\text { Percent correct: } \sim 0 \%\end{array}$ \\
\hline Alternation & $\begin{array}{c}\text { Latency: similar to PM } \\
\text { Percent correct: } ~ 0 \%\end{array}$ & $\begin{array}{c}\text { Latency: similar to AM } \\
\text { Percent correct: } \sim 0 \%\end{array}$ \\
\hline
\end{tabular}

Figure 1. Behavior of rats on Skip AM and Skip PM sessions if they are using alternation, ordinal, or circadian timing. For example, if the rat was using an ordinal timer, it would be expected to behave as though it were the morning following both Skip AM and Skip PM probe sessions.
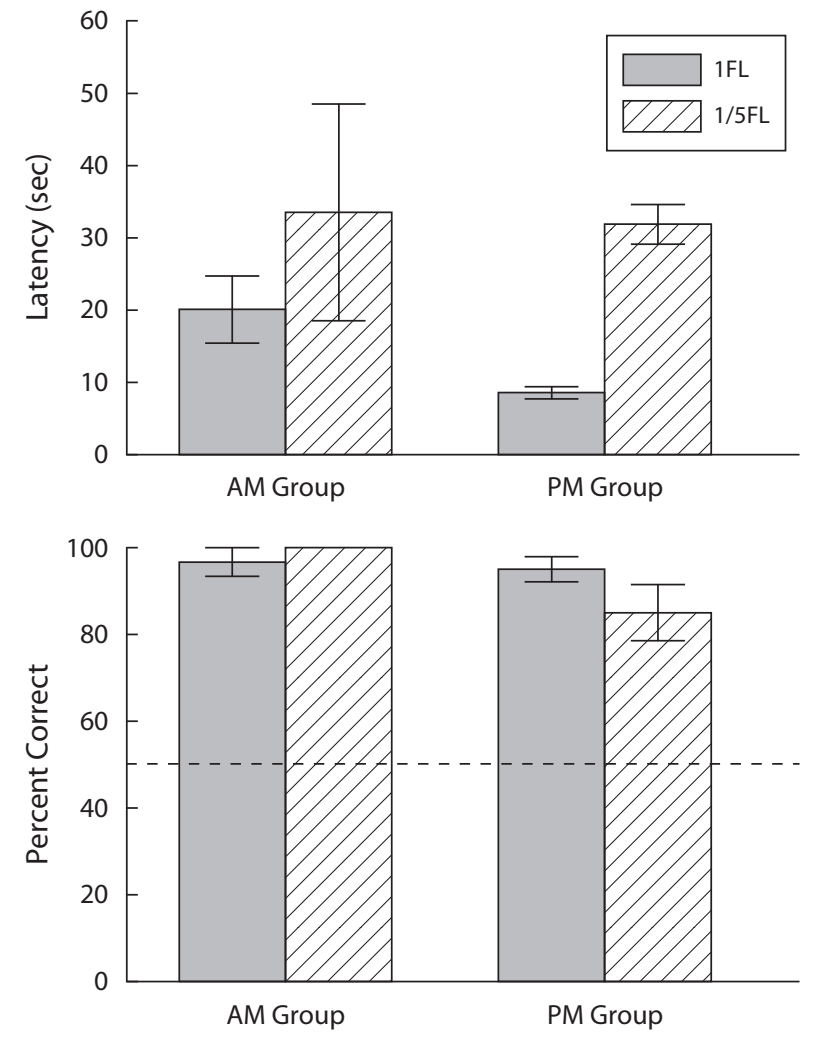

Figure 2. Average latency to leave the start arm and percent correct arm choices for the $\mathbf{1 0}$ days prior to the start of probe sessions on sessions with 1FL and 1/5FL. The horizontal dashed line represents chance. (Note that there is no error bar for the AM group's percent correct in the $1 / 5 \mathrm{FL}$ condition because there was no variance.)

to the start of probe sessions. A 2 within (amount: 1FL vs. $1 / 5 \mathrm{FL}) \times 2$ between (group: AM vs. PM) ANOVA showed that the latencies were significantly faster on trials with more food than on trials with less food and that the PM group was significantly faster than the AM group [amount, $F(1,5)=16.154, p=.010$; amount $\times$ group, $F(1,5)=1.165, p=.330$; group, $F(1,5)=31.189, p=$ $.003]$. This significant difference between the two groups may have been due to the PM group being more hungry. There was also a significant difference between the groups on the percent correct choices [group, $F(1,5)=8.929$, $p=.031$; amount, $F(1,5)=0.357, p=.576$; amount $\times$ group, $F(1,5)=1.429, p=.286]$. According to the binomial distribution, a given rat would be significantly above chance if it correctly chose the correct arm at least 15 out of the 20 sessions. This was true for all 7 rats, with the lowest percent correct choice score equal to 17 out of 20 . This corresponds to a $p$ value of .001 .

To determine how the rats learned the daily TPL task, the training period was broken down into 13 blocks of 10 days each. Only the first 13 blocks were examined, because in Block 14 the first rat began probe testing. Figure 3 shows the average latency and percent correct choices for each of the first 13 blocks of training. Looking first at the latency data, we can see that at the start of training the rats 

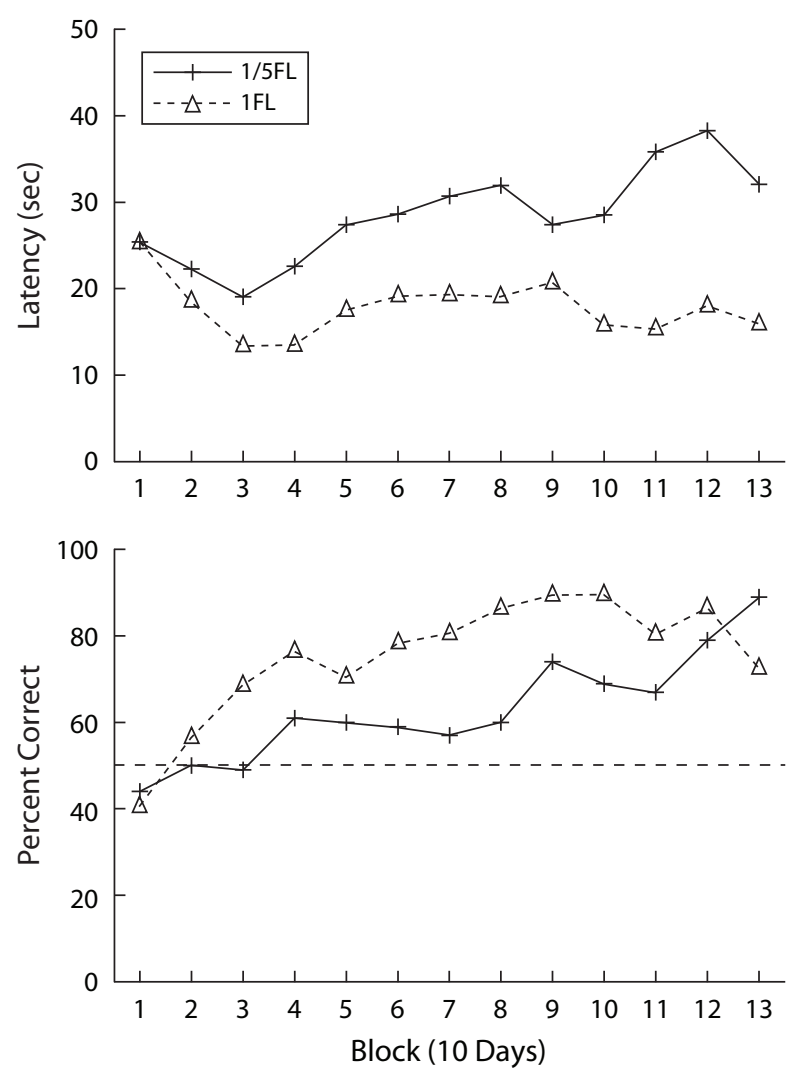

Figure 3. Average latency and percent correct choices for the first 13 blocks of training for sessions with 1FL and 1/5FL. The horizontal dashed line represents chance.

had similar latencies on both the $1 \mathrm{FL}$ and $1 / 5 \mathrm{FL}$ sessions; however, as the training continued, the latencies quickly diverged, with reduced latencies on the sessions with more food. To confirm these observations statistically a 2 within (amount) $\times 13$ within (block) $\times 2$ between (group) repeated measures ANOVA was conducted. There was no main effect of group $[F(1,5)=0.010, p=.924]$, nor were any of the interactions involving group significant. There was a significant amount main effect $[F(1,5)=8.755, p=$ $.032]$ and a near significant block main effect $[F(12,60)=$ $1.773, p=.074]$. There was also a significant amount $\times$ block interaction $[F(12,60)=3.741, p<.001]$. Because there was no significant difference between the two groups, we averaged across the groups and conducted paired sample $t$ tests as follow-ups. This analysis showed a nonsignificant difference between the two types of sessions on Block $1[t(6)=-.033, p=.975]$, but by Block 2 the difference between the two types of sessions was significantly different $[t(6)=2.495, p=.047]$. The difference between the two session types remained significant for Blocks 3-13 ( $p$ s ranging from .013 to .049).

To examine how the percent correct scores (Figure 3, bottom panel) varied across blocks and session types, a 2 within (amount) $\times 13$ within (block) $\times 2$ between (group) repeated measures ANOVA was conducted. There was no main effect of group $[F(1,5)=0.511, p=.507]$ nor were any of the interactions involving group significant. It was found that the percent correct choices were significantly different across blocks $[F(12,60)=8.731, p<.001]$. However, there was no significant amount $[F(1,5)=1.192, p=$ $.325]$ nor amount $\times$ block interaction $[F(12,60)=1.288$, $p=.249$ ] effect. We were also interested in determining at what point the percent correct choices differed significantly from chance $(50 \%)$. To determine this, 26 one-sample $t$ tests were conducted with scores pooled across the two groups. Table 1 shows the results of these $t$ tests. To help minimize the probability of Type I error, we set significance at .01. The chance of Type I error was also reduced by the fact that we were looking at a pattern of results (i.e., whether the data were consistent block by block). On the $1 / 5 \mathrm{FL}$ sessions, the percent of correct choices was not consistently above chance until Block 12, whereas on the 1FL sessions the percent of correct choices was consistently above chance starting at Block 6 . These results suggest that the rats learned the task faster under the $1 \mathrm{FL}$ condition than under the $1 / 5 \mathrm{FL}$ condition.

To determine which type of timer the rats were using, we examined the latency and percent correct choices for the baseline and probe sessions. Because previous research (Thorpe et al., 2003) has shown that there are individual differences in the timing strategies used by rats in time-ofday discrimination tasks, we decided to first look at the data for each rat rather than averaging across rats. The baseline and probe data for each rat are shown in Figure 4. Visual inspection of the latency data shows a similar pattern of results for all rats, namely that the Skip PM latencies were similar to the AM latencies, and the Skip AM latencies were similar to the PM latencies. These results suggest that all rats used a circadian strategy. The binomial probability of all 7 rats showing an identical pattern of latencies is .0078 . The percent correct data, on the other hand, were inconclusive regarding the timing mechanism employed.

Since all of the rats demonstrated the same pattern of results, group analyses were conducted to confirm the hypothesis that the rats were using circadian timing. Average latencies and percent correct choices were computed for the baseline sessions interspersed within the probes, as

Table 1

Results of One-Sample $t$ Tests Comparing Percent Correct Choices on Sessions with 1/5FL and 1FL on the Training Session to Chance Levels of $50 \%$

\begin{tabular}{crrrrr}
\hline & \multicolumn{2}{c}{$1 / 5 \mathrm{FL}$} & & \multicolumn{2}{c}{$1 \mathrm{FL}$} \\
\cline { 2 - 3 } \cline { 5 - 6 } Block & $t$ & \multicolumn{1}{c}{$p$} & & \multicolumn{1}{c}{$t$} & \multicolumn{1}{c}{$p$} \\
\hline 1 & -.620 & .558 & & -0.938 & .384 \\
2 & -.001 & $>.999$ & & 0.507 & .630 \\
3 & -.110 & .916 & & .167 & .073 \\
4 & 1.082 & .321 & 9.500 & $<.001$ \\
5 & -.851 & .427 & & 2.905 & .027 \\
6 & .724 & .496 & & 10.954 & $<.001$ \\
7 & .591 & .576 & & 22.000 & $<.001$ \\
8 & .866 & .420 & 7.120 & $<.001$ \\
9 & 1.972 & .096 & 10.583 & $<.001$ \\
10 & 1.518 & .180 & & 18.330 & $<.001$ \\
11 & 2.295 & .061 & 6.181 & .001 \\
12 & 5.620 & .001 & 13.000 & $<.001$ \\
13 & 6.971 & $<.001$ & 4.042 & .007 \\
\hline
\end{tabular}

Note-In all cases, $d f=6$. FL, Froot Loop. 

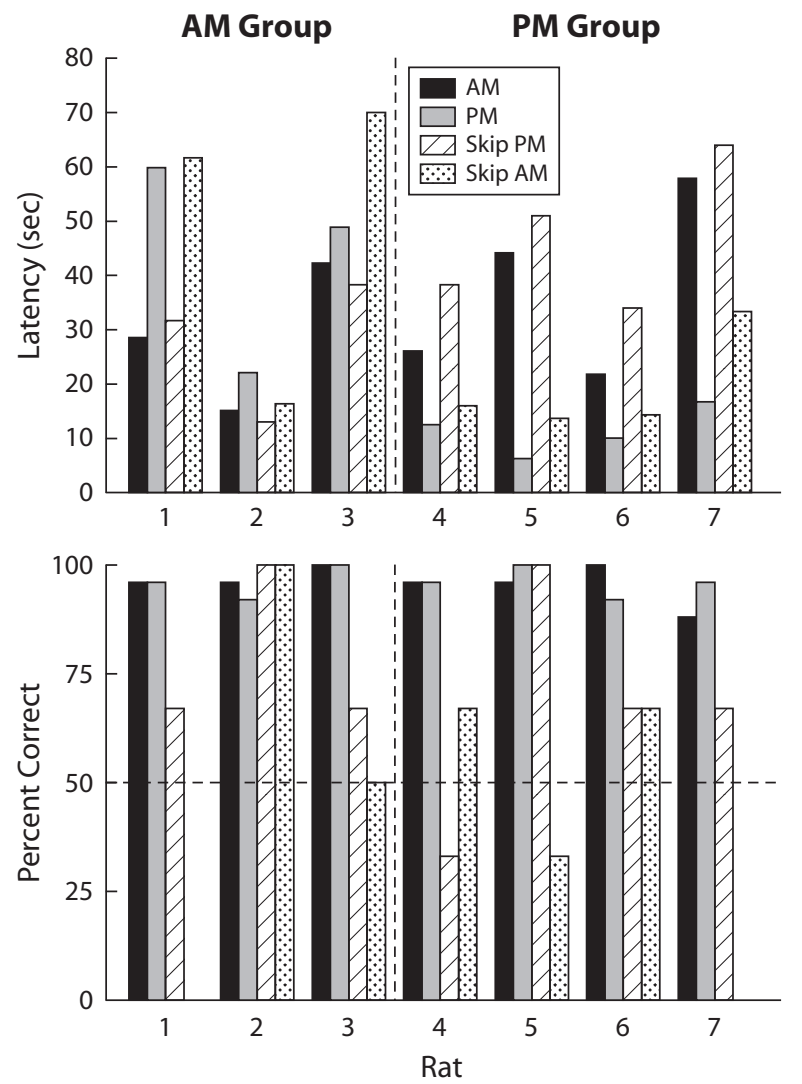

Figure 4. Latency and percent correct scores for the baseline (based on the averages for the 4 days prior to the probes) and skip probe data for each rat. AM Group refers to the rats that received $1 \mathrm{FL}$ in morning sessions and 1/5FL in afternoon sessions. PM Group refers to the rats that received 1FL in afternoon sessions and 1/5FL in morning sessions. The horizontal dashed line represents chance. (Note in some instances, e.g., percent correct on Skip AM for Rat 1, there is no bar shown. This is because the percent correct was 0 .)

well as for the probes themselves. The average baseline scores were based on the averages for the 4 days prior to the probes (i.e., 24 trials for all rats, except Rat 3, who had 20 trials). These averages are shown in Figure 5.

To confirm that rats were using a circadian timing strategy, four conditions had to be met with regard to the latency data (see Figure 1). First, the Skip PM latencies should not be significantly different from the AM baseline. Second, the Skip PM latencies should be significantly different from the PM baseline: For the AM group, the Skip PM latency should be less than the PM baseline, and for the PM group, the Skip PM latency should be greater than the PM baseline. Third, the Skip AM latencies should be significantly different from the AM baseline: For the AM group, the Skip AM latency should be greater than the AM baseline, and for the PM group, the Skip AM latency should be less than the AM baseline. And finally, the Skip AM latencies should not be significantly different from the PM baseline.

To test these conditions, four 2 within (type: probe vs. baseline) $\times 2$ between (group) ANOVAs were conducted comparing the relevant latencies. In the first case (comparison of Skip PM and AM baseline latencies), a significant type $\times$ group interaction $[F(1,5)=15.316, p=$ $.011]$ suggested that at least one of the groups differed in their latency between Skip PM and AM baseline. Simple main effects analyses demonstrate that this difference is in the PM group $[F(1,5)=29.142, p=.003]$ but that the AM group $[F(1,5)=0.252, p=.637]$ conformed to the hypotheses (see Figure 5, top panel). To better understand the significant difference found between the Skip PM and baseline AM latencies for the PM group, we should examine Figure 5 more carefully. The latency on the Skip PM session was actually greater than that on the AM baseline. If the rats were using an alternation strategy, we would have expected the latency to be more similar to the baseline PM data. The comparison of the Skip PM and baseline AM latencies supported the first condition of circadian timing.

The other three tests conformed more straightforwardly with our hypotheses. A significant type $\times$ group interaction $[F(1,5)=33.377, p=.002]$, combined with simple main effects, revealed that the PM group significantly differed between Skip PM and PM baseline latencies $[F(1,5)=37.040, p=.002]$ but that the AM group trended toward the same difference, but in the opposite direction $[F(1,5)=5.626, p=.064]$. Similarly, a significant type $\times$ group interaction $[F(1,5)=13.531, p=$ .014] was found when testing the third condition (Skip AM and AM baseline). Simple main effects conducted as follow-up found both the AM group $[F(1,5)=6.708$, $p=.049]$ and the PM group $[F(1,5)=6.908, p=.047]$ demonstrated statistically significant differences, but these differences, consistent with our hypotheses, were in opposing directions. And finally, the fourth condition for circadian timing (Skip AM and PM baseline) demonstrated no significant differences [type, $F(1,5)=3.248$, $p=.131$; type $\times$ group, $F(1,5)=0.087, p=.780$; group, $F(1,5)=6.639, p=.050]$.

A similar series of analyses was conducted comparing the percent correct choices on the probe and baseline sessions. The results of these analyses were inconclusive, similar to those found when the individual data were examined. There was a difference of marginal significance in the percent correct on the Skip PM, compared with both the AM baseline [type, $F(1,5)=6.158, p=.056$; type $\times$ group, $F(1,5)=0.205, p=.670$; group, $F(1,5)=0.530$, $p=.499$ ] and the PM baseline [type, $F(1,5)=6.303, p=$ .054 ; type $\times$ group, $F(1,5)=0.349, p=.580$; group, $F(1,5)=0.349, p=.580]$. The percent correct on the Skip AM were significantly different from both the AM baseline [type, $F(1,5)=11.920, p=.018$; type $\times$ group, $F(1,5)=$ $0.042, p=.845$; group, $F(1,5)=0.111, p=.752]$ and the PM baseline [type, $F(1,5)=9.726, p=.026$; type $\times$ group, $F(1,5)=0.069, p=.804$; group, $F(1,5)=0.082$, $p=.786]$. These results do not correspond to the predictions shown in Figure 1 if a circadian or ordinal timer or a nontiming alternation strategy were used. Rather than follow the predictions of any model, it would appear that 

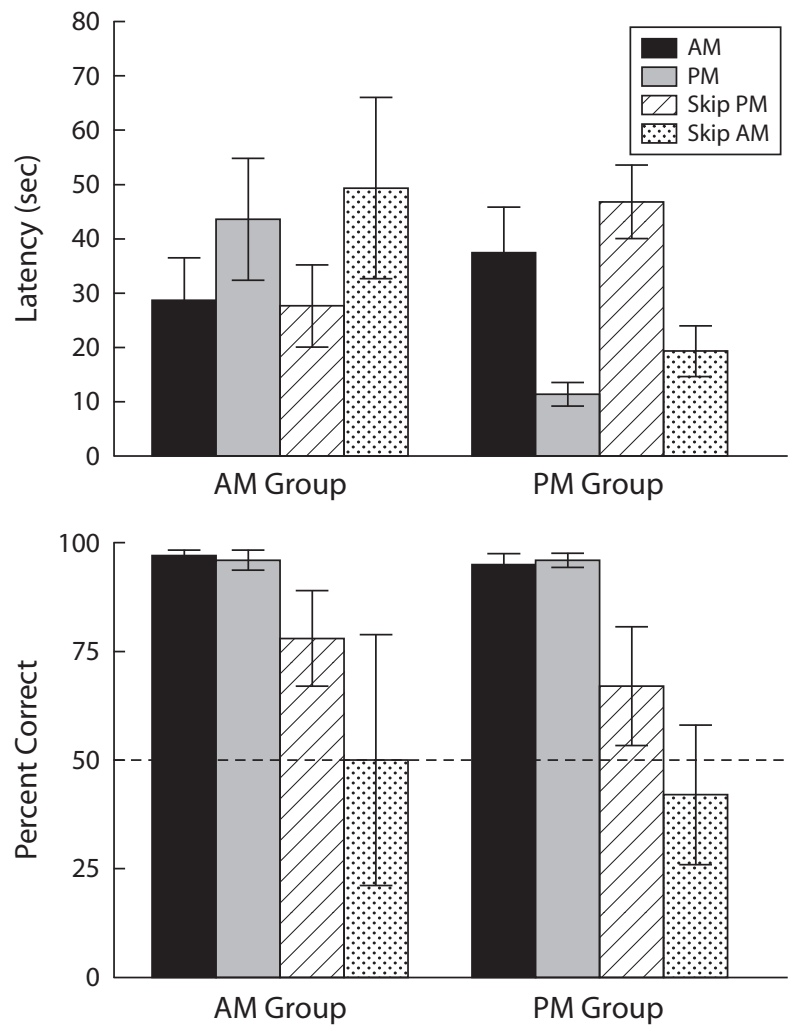

Figure 5. Average latencies and percent correct scores for the skip probe sessions and for the baseline sessions (based on the averages for the 4 days prior to the probes) interspersed within the probes. AM Group refers to the rats that received $1 \mathrm{FL}$ in morning sessions and $1 / 5 \mathrm{FL}$ in afternoon sessions. PM Group refers to the rats that received $1 \mathrm{FL}$ in afternoon sessions and $1 / 5 \mathrm{FL}$ in morning sessions. The horizontal dashed line represents chance.

the rats were simply responding at chance levels. This was tested using $t$ tests comparing the Skip AM and Skip PM for both groups to the chance level of $50 \%$. It was found that in all cases the percent correct on the skip probe tests was not significantly different from chance [AM group, Skip PM, $t(2)=2.500, p=.130$; and Skip AM, $t(2)=$ $0.000, p>$.999; PM group, Skip PM, $t(3)=1.225, p=$ .308 ; and Skip AM, $t(3)=-0.527, p=.635]$.

\section{DISCUSSION}

In the present study, rats were trained on a daily TPL task in which the amount of food varied with the spatiotemporal contingencies. During training it was found that rats quickly learned to go faster on the trials with $1 \mathrm{FL}$ than on the trials with only $1 / 5 \mathrm{FL}$. In fact, by the second block of 10 days this latency difference between the two types of sessions was significantly different. However, it was not until Block 6 that the rats appeared to have learned which arm was associated with the food at which time of day. The percent correct responses reached significantly different levels from chance in the trials with 1FL around Block 6, whereas they did not reach statistically signifi- cantly different levels until Block 12 on the trials with only $1 / 5 \mathrm{FL}$. It would thus appear that the rats learned the time of day that was associated with a particular amount of food prior to learning where the food was located.

These results are important, given that they are the first clear evidence of learning in a low-response-cost daily TPL task. Using an almost identical procedure, Means and colleagues (Means, Arolfo, et al., 2000; Means, Ginn, et al., 2000) showed only a moderate amount of learning. They also used a T-maze with food in one location in the morning and in another location in the afternoon. However, in their design the same amount of food was used in all sessions. Neither making the two places more distinctive nor making the two times farther apart during the day significantly increased rats' success rate on the task. In addition, increased training and minimization of external cues led to decreases in performance. In contrast, in the present study the rats readily learned the task by simply giving differential amounts of food on morning and afternoon trials.

These results are reminiscent of those in the differential outcomes in discrimination learning literature. Urcuioli (2005), in a recent review, stressed that reinforcers are just as important as the stimuli and responses in discrimination learning. He cited the earlier work of Trapold (1970), in which rats were trained to press one lever in the presence of one auditory stimulus and another lever in the presence of a different auditory signal. If sucrose water was paired with one auditory signal/lever and food with the other auditory signal/lever, the rats learned more quickly than if both auditory signals/levers had been paired with the same reinforcer. On the basis of this finding, as well as on a review of the literature, it was suggested that in the differential outcome paradigms the rats learn to press one lever when expecting sucrose water and the other lever when expecting food.

The differential outcome literature can provide insight into how the rats in the present study learned the daily TPL task, whereas rats trained in a similar task with the same amount of food in both sessions did not. Very early in their training, the rats learned to expect different amounts of food at different times of day. This can be seen by the fact that the latencies on trials with $1 \mathrm{FL}$ were significantly less than on trials with $1 / 5 \mathrm{FL}$ by Block 2 . The rats then learned to go to one location when $1 \mathrm{FL}$ was expected and to go to another location when $1 / 5 \mathrm{FL}$ was expected. However, the rats did not seem to learn the locations until much later: Block 6 for the 1FL trials, and Block 12 for the 1/5FL trials. Rather than learning to associate time, place, and event, these rats seemed to have learned an association first between time and event (i.e., reinforcer) and then between event and place.

As outlined in the introduction, there are various studies showing the difficulties that rats have in learning daily TPL tasks. Currently, the best hypothesis to explain why rats learn some TPL tasks but not others is the responsecost hypothesis put forth by Widman et al. (2000), but this hypothesis cannot explain the present data or some of the other inconsistencies summarized in the introduction. One thing that is clear, however, is that Gallistel's (1990) 
tripartite theory needs to be revised. Rats do not readily learn time-place discriminations; this would argue against Gallistel's idea that tripartite codes are automatically encoded with the nature of the event and the time and place in which the event occurred.

We would like to put forth an alternative hypothesis to that of Gallistel's (1990) tripartite theory. We propose that animals encode spatiotemporal information in a series of bipartite codes that can later become associated with one another. We argue that when an animal is faced with a biologically important event, bipartite codes are automatically formed. These bipartite codes are time-event and event-place. Whereas spatiotemporal information is typically stored in bipartite codes, under some circumstances, such as those with a high response cost, tripartite time-event-place codes may be formed.

To support our bipartite hypothesis, we would like to consider the results of various TPL tasks that have been investigated to date. Figure 6 summarizes the memory codes and decision processes involved in four different types of TPL tasks. Starting with the present study of differential food as the first example, shown in panel A, the bipartite memory codes would be in the forms time-event and event-place. Consider a rat that received a $1 / 5 \mathrm{FL}$ in the left arm in morning sessions and 1FL in the right arm in afternoon sessions: Its memory codes would be time ${ }_{1}-$ event $_{1 / 5 \mathrm{FL}} ;$ time $_{2}-$ event $_{1 \mathrm{FL}} ;$ event $_{1 / 5 \mathrm{FL}}-$ place $_{1}$; event $_{1 \mathrm{FL}}-$ place $_{2}$. In morning sessions, the time ${ }_{1}-$ event $_{1 / 5 \mathrm{FL}}$ code is drawn on. Searching through the memory codes, it also finds that event ${ }_{1 / 5 \mathrm{FL}}$ has been associated with place ${ }_{1}$ and therefore in the morning sessions it goes to place ${ }_{1}$. Likewise, the rat learns to go to place ${ }_{2}$ at time $e_{2}$.

Rats have been shown to successfully learn time-ofday discriminations using a go-no go design (Means, Arolfo, et al., 2000; Thorpe et al., 2003, Experiment 5). Figure 6B shows how the bipartite hypothesis might explain these data. Using the example of a rat that receives food in morning sessions in both the arms but no food in either arm in afternoon sessions, the memory codes would be time ${ }_{1}-$ event $_{\text {food }} ;$ time $_{2}-$ event $_{\text {no food }}$; event food $_{\text {flace }}$; event $_{\text {food }}-$ place $_{2} ;$ event $_{\text {no food }}-$ place $_{1} ;$ event $_{\text {no food }}-$ place $_{2}$. In morning sessions, a search of the time-event codes would find that time ${ }_{1}$ was associated with event ${ }_{\text {food }}$, and the rat's latency to leave the start arm will be short (at time ${ }_{2}$ the association is with event $t_{\text {no food }}$ and as such the rat's latency to leave the start arm will be longer). The results of both studies confirm this hypothesis. (Note that both places are associated equally with food and with no food; therefore, the rats go to both locations on all trials.)

The typical low-response-cost daily TPL task in which the same amount of food is given in one location in morning sessions and in another location in afternoon sessions

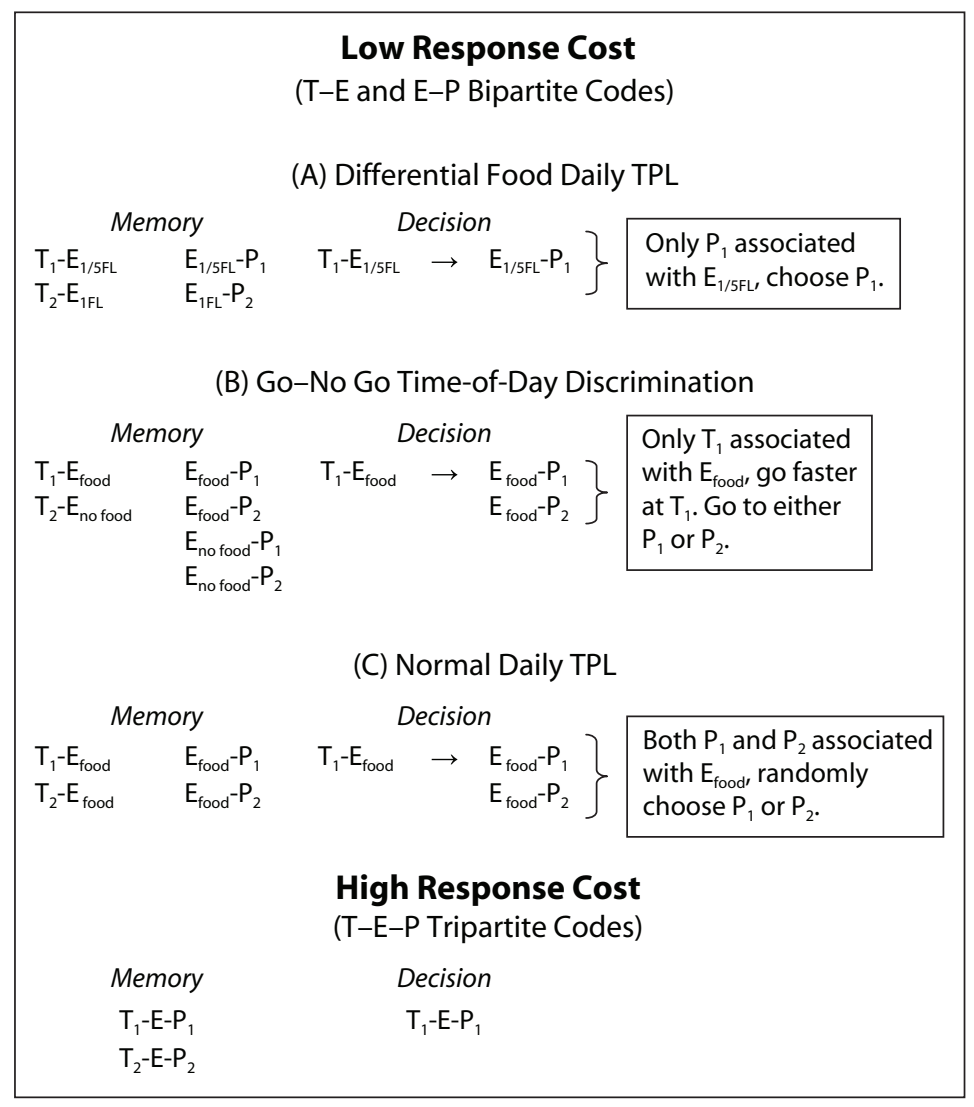

Figure 6. Memory codes and decision processes involved in various types of TPL tasks, on the basis of a bipartite hypothesis. T, time; E, event; P, place; TPL, time-place learning. 
is illustrated in panel $\mathrm{C}$ of Figure 6. The memory codes in this situation are time ${ }_{1}$ - event $_{\text {food }} ;$ time $_{2}$ - event $_{\text {food }}$; event $_{\text {food }}$ place $_{1} ;$ event $_{\text {food }}-$ place $_{2}$. In morning sessions, the rat consults its memory codes and determines that time ${ }_{1}$ is associ-

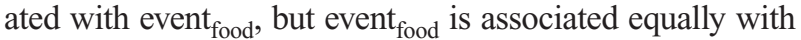
both place ${ }_{1}$ and place $_{2}$. Therefore, in morning (and afternoon) sessions the rat goes to place $150 \%$ of the trials and to place $_{2} 50 \%$ of the trials. This explains the place preference (but no TPL learning) that is seen in low-response-cost daily TPL tasks (Means, Arolfo, et al., 2000; Means, Ginn, et al., 2000; Thorpe et al., 2003).

Finally, panel $\mathrm{C}$ of Figure 6 shows the hypothesized memory codes and decisions in high-response-cost daily TPL tasks. In high-response-cost tasks, we suspect that a tripartite code is formed that includes the time, place and event. If the rat receives food in one location of a climbing maze in the morning, on morning sessions it can consult its memory codes to find that food is only ever associated in that place at that time.

Although it has now been shown that rats are capable of learning a low-response-cost daily TPL task, the question of what type of timer is being used still remains. As discussed in the introduction, there are three likely possibilities: circadian, ordinal, and a nontiming alternation strategy. (However, see Pizzo \& Crystal, 2006, for an argument for the use of interval timing in some daily TPL tasks.) To determine which type of timer was being used, skip session probes were conducted. On the probe tests, the latency to leave the start arm and the percent correct choices made were analyzed. Both the individual data and the average data for the latencies suggested that the rats were using a circadian strategy. The results for the percent correct responses were inconclusive. However, these inconclusive results could be explained by the bipartite hypothesis. If the rats are encoding the time-event and event-place information in separate codes, the location of the food (i.e., percent correct choices) is not directly associated with the time of day. Thus, it is not surprising that the percent correct choices cannot distinguish which timing strategy is being used.

\section{AUTHOR NOTE}

This research was supported by the Natural Sciences and Engineering Research Council of Canada (NSERC). We thank Daniela Meier, Jessica Otte, Siu Him Chan, and Angeles Barrera for help with running the studies and Darcy Hallett for assistance with statistical analyses. Send all correspondence relating to this article to C. M. Thorpe, Department of Psychology, University College London, Gower Street, London WC1E 6BT, England (e-mail: christina.thorpe@ucl.ac.uk).

\section{REFERENCES}

Biebach, H., Gordin, M., \& Krebs, J. R. (1989). Time-and-place learning by garden warblers, Sylvia borin. Animal Behaviour, 37, 353-360.
Boulos, Z., \& Logothetis, D. E. (1990). Rats anticipate and discriminate between two daily feeding times. Physiology \& Behavior, 48, 523-529.

CARr, J. A. R., \& Wilkie, D. M. (1997a). Ordinal, phase, and interval timing. In C. M. Bradshaw \& E. Szabadi (Eds.), Time and behaviour: Psychological and neurobehavioural analyses (pp. 265-327). Amsterdam: Elsevier.

CARR, J. A. R., \& Wilkie, D. M. (1997b). Rats use an ordinal timer in a daily time-place learning task. Journal of Experimental Psychology: Animal Behavior Processes, 23, 232-247.

CARR, J. A. R., \& WILKIE, D. M. (1999). Rats are reluctant to use circadian timing in a daily time-place task. Behavioural Processes, 44, 287-299.

Gallistel, C. R. (1990). The organization of learning. Cambridge, MA: MIT Press.

Means, L. W., Arolfo, M. P., Ginn, S. R., Pence, J. D., \& Watson, N. P. (2000). Rats more readily acquire a time-of-day go no-go discrimination than a time-of-day choice discrimination. Behavioural Processes, 52, 11-20.

Means, L. W., GinN, S. R., Arolfo, M. P., \& Pence, J. D. (2000). Breakfast in the nook and dinner in the dining room: Time-of-day discrimination in rats. Behavioural Processes, 49, 21-33.

Mistlberger, R. E., De GroOT, M. H. M., Bossert, J. M., \& MarCHANT, E. G. (1996). Discrimination in circadian phase in intact and suprachiasmatic nuclei-ablated rats. Brain Research, 739, 12-18.

Pizzo, M. J., \& Crystal, J. D. (2002). Representation of time in timeplace learning. Animal Learning \& Behavior, 30, 387-393.

PIZZo, M. J., \& CRYSTAL, J. D. (2004). Evidence for an alternation strategy in time-place learning. Behavioural Processes, 67, 533-537.

Pizzo, M. J., \& Crystal, J. D. (2006). The influence of temporal spacing on time-place discrimination. Learning \& Behavior, 34, 131-143.

ReEbs, S. G. (1993). A test of time-place learning in a cichlid fish. Behavioural Processes, 30, 273-282.

REEBS, S. G. (1999). Time-place learning based on food but not on predation risk in a fish, the inanga (Galaxias maculatus). Ethology, 105, 361-371.

SAKsidA, L. M., \& WiLKIE, D. M. (1994). Time-of-day discrimination by pigeons, Columba livia. Animal Learning \& Behavior, 22, 143-154.

Thorpe, C. M., Bates, M. E., \& Wilkie, D. M. (2003). Rats have trouble associating all three parts of the time-place-event memory code. Behavioural Processes, 63, 95-110.

THORPE, C. M., \& WILKIE, D. M. (2006). Properties of time-place learning. In T. R. Zentall \& E. A. Wasserman (Eds.), Comparative cognition: Experimental explorations of animal intelligence (pp. 229-245). Oxford: Oxford University Press.

Timberlake, W., \& White, W. (1990). Winning isn't everything: Rats need only food deprivation and not food reward to traverse a radial arm maze. Learning \& Motivation, 21, 153-163.

TRaPold, M. A. (1970). Are expectancies based upon different positive reinforcing events discriminably different? Learning \& Motivation, 1, 129-140.

URCUIOLI, P. J. (2005). Behavioral and associative effects of differential outcomes in discrimination learning. Learning \& Behavior, 33, 1-21.

WAHL, O. (1932). Neue Untersuchungen über das Zeitgedächtnis der Bienen. Zeitschrift für Vergleichende Physiologie, 16, 529-589.

Widman, D. R., Gordon, D., \& Timberlake, W. (2000). Response cost and time-place discrimination by rats in maze tasks. Animal Learning \& Behavior, 28, 298-309.

Widman, D. R., Sermania, C. M., \& Genismore, K. E. (2004). Evidence for time-place learning in the Morris water maze without food restriction but with increased response cost. Behavioural Processes, 67, 183-193.

(Manuscript received September 7, 2006; revision accepted for publication December 4, 2006.) 